\title{
EDITORIAL
}

\section{Critical care journals during the COVID-19 pandemic: challenges and responsibilities}

\author{
Giuseppe Citerio ${ }^{1 *} \mathbb{D}$, Jan Bakker ${ }^{2,3,4}$, Laurent Brochard ${ }^{5}$, Timothy G. Buchman 6 , Samir Jaber ${ }^{7}$, Peter J. Mazzone ${ }^{8}$, \\ Jean-Louis Teboul ${ }^{9}$, Jean-Louis Vincent ${ }^{10}$ and Elie Azoulay ${ }^{11}$
}

C 2020 Springer-Verlag GmbH Germany, part of Springer Nature

COVID-19 has had a profound impact on the critical care community, as one of the front-line areas in the ongoing pandemic, and on its journals. In response to the COVID-19 emergency, 'observational research' is being produced at an unprecedented rate. Although randomized trials were initially in the minority, recently more than 500 clinical trials have been formally registered. Consequently, medical journals have been overwhelmed with manuscripts of all types, mostly observational, and often anecdotal and in short format. All critical care journals have recorded a huge increase in the number of submissions in the first quarter of this year compared with the same period in 2019. Clinicians, justifiably, have been eager to get information about this frightening new disease, while the lay press has put COVID-19 developments in the spotlight, often without distinguishing between fake news, anecdotes and solid science. The various social media, as usual, have acted as an amplifier of what seems to be a phenomenon unprecedented in the history of modern medicine.

A Medline search for COVID-19-related literature (May 26, 2020) returned more than 15,500 published papers, including 1,870 in the critical care field. In facing the current situation, we critical care journal editors have been trying to balance the need for fast and easy access to new knowledge with our commitment to publishing quality data and unbiased results. We have also been striving to detect research misconduct and violation of publication ethics. During this pandemic, we have

\footnotetext{
${ }^{*}$ Correspondence: giuseppe.citerio@unimib.it

${ }^{1}$ Editor in Chief, Intensive Care Medicine. Editor in Chief, Intensive Care

Medicine Experimental, School of Medicine and Surgery, University of Milano - Bicocca, Monza, Italy

Full author information is available at the end of the article
}

felt a particular need to maintain, and indeed underline, our primary responsibility, which is to bring appropriate knowledge to the bedside and hopefully improve ICU practices. The pressures brought by the current, unprecedented, pandemic have forced researchers to act quickly in order to save lives; however it remains imperative that research continue to adhere to the guiding concepts, albeit sometimes imperfect, of medical research.

We editors of critical care journals have felt particular pressure, but also a specific responsibility, with regard to this endeavor. We therefore wish to raise, and share with readers, the following points:

\section{Ethics of research}

The ethics of any research begin with the start of that research at the different sites. In this respect, institutional review boards (IRBs) play their part by reviewing proposed studies and maintaining oversight. However, the current pandemic, with the sheer number of studies submitted, has put pressure on this system. Due to the lack of known mechanistic processes involved in the severity of the disease, and the fact that no treatment is yet available, there has emerged a need for an expedited review process for submitted studies, together with continued adequate oversight of active studies. Most of these aspects are the responsibility of local/national IRBs, but editors, too, need to remain committed to upholding the rules of ethical research in their journals, including the ongoing need for discussion of the consent process.

\section{Appropriate study design}

The COVID-19 epidemic has presented the leading journals with major challenges in terms of deciding what constitutes an acceptable study design. Aiming to disseminate knowledge, the first reports focused mainly on

\section{Springer}


direct experience and small case series. We understand this initial choice, to publish experience and the results of small studies, because in the early stages of this extraordinary pandemic, there were still a lot of unknowns. However, editors have nevertheless remained vigilant about study design and the external validity of results, and as knowledge has increased over time, they have become more selective, focusing more on classic research designs, with ethical justification, and the inclusion of coherent standards of care or usual care arms.

\section{Fair and speedy peer review to ensure quality of science}

Editors are responsible for ensuring fair, adequate and speedy reviews of studies at all times. The current epidemic has put severe strain on this system, too, as not only many editors but also reviewers have been kept busy handling the epidemic. This has increased the rate of desk-rejects (in many cases to more than $60 \%$ of submissions). The rush to disseminate knowledge in this pandemic has put the review process under considerable pressure to shorten the time from submission to online publication. Editors have the responsibility to ensure adequate consultation of experts in the field, which must be balanced with the perceived importance and implications of the results. This consultation process has been undermined by the exponential increase in preprint publications (studies published on free-access archives/sites with only minimal review and no peer review). Appeals [1] to share research data and findings relevant to the COVID-19 outbreak are driving an unprecedented surge in manuscripts posted on open-source platforms related to the pandemic. By May 2020, bioRxiv and medRxiv had already posted almost 3000 COVID-related papers. This rush to publish information without adequate peer review has led to the publication of studies that ultimately had to be retracted [2].

This latter situation carries major risks since rapidly released but inadequately scrutinized data may be perceived as valid study results, and therefore induce changes in clinical practice. Unfortunately, some of these publications have been covered by mainstream media. Editors have the difficult duty to balance the desire for pre-publication with the importance of an adequate peerreview process. We think it will be essential, in the future, to generate a framework to deal with this problem.

\section{Fast publication}

Electronic communication has undoubtedly made the publication process much faster than in the past. Following a review process, which must be as rapid as possible, publishers as well as authors should strive to edit their proofs within the shortest possible time. Once a manuscript has been accepted, the time to publication can be improved by initially publishing the unedited PDF, and subsequently producing a fully edited galley proof to be published on-line first. In this way, journals belonging to teaching societies, such as the European Society of Intensive Care Medicine (ESICM), the American Thoracic Society (ATS), the American College of Chest Physicians (ACCP), and the Society of Critical Care Medicine (SCCM), have been able to publish provisionally accepted PDFs in less than $24 \mathrm{~h}$. This method allows readers access only to peer-reviewed reports, and thus reduces the risk of diffusing misleading, and sometimes incorrect, information.

\section{No paywalls}

Publishers have reacted to the pandemic by offering open-access platforms to facilitate fast and direct access to the latest available research. To date, many publishers have agreed to make their COVID-19 papers freely available as requested by the Welcome Trust. Most journals have removed paywalls for COVID-19 articles, at least for the duration of the outbreak.

\section{New methods for transferring knowledge}

Even in pandemics, editors should ensure publication of reliable data with adequate translation to clinical practice where indicated. Once the review process has finished, early notification of accepted manuscripts and their diffusion on social media are ways to disseminate the knowledge rapidly. Editors and authors have a responsibility to promote new methods, such as author interviews and webinars with scientific societies, that can allow correct knowledge to be disseminated not only to the general public, but also to the numerous colleagues who lack the time to keep up with all the new data. Also, these new means of communication can play an important role in preventing circulation of unproven strategies and misinterpretations.

We, as critical care journal editors, need to be aware that the critical care community relies on the quality assurance and curating work done by our scientific journals. Research in the current pandemic has been marred by poor study designs, uncontrolled interventions, unchecked data and suboptimal statistical analyses [3]. Editors should safeguard the use of proper scientific methods and reporting while guiding post-release interpretations through editorials where required.

What will remain after this crisis? The world will be changed. We hope that scientific journals, with their imperfect but rigorous and fair review processes, will have reached even a higher standard for their readers, 
proving to be the right place to find, in a reasonable amount of time-depending on the urgency of the situation, appropriate clinical science for patients.

\author{
Author details \\ ${ }^{1}$ Editor in Chief, Intensive Care Medicine. Editor in Chief, Intensive Care \\ Medicine Experimental, School of Medicine and Surgery, University of Milano \\ - Bicocca, Monza, Italy. ${ }^{2}$ Editor in Chief, Journal of Critical Care, NYU School \\ of Medicine, Columbia University College of Physicians \& Surgeons, New \\ York, USA. ${ }^{3}$ Erasmus MC University Medical Center Rotterdam, Rotterdam, \\ The Netherlands. ${ }^{4}$ Pontificia Universidad Católica de Chile, Santiago, Chile. \\ ${ }^{5}$ Deputy Editor, American Journal of Respiratory and Critical Care Medicine, \\ Keenan Research Centre, St Michael's Hospital and Interdepartmental Division \\ of Critical Care Medicine, University of Toronto, Toronto, ON, Canada. ${ }^{6}$ Editor \\ in Chief, Critical Care Medicine, Emory Critical Care Center, Emory University, \\ Atlanta, Georgia. ${ }^{7}$ Deputy Editor, Intensive Care Medicine, Département \\ Anesthésie et Réanimation B (DAR B), Centre Hospitalier Universitaire (CHU) de \\ Montpellier, Hôpital Saint-Éloi, and INSERM U-1046, Montpellier, France. ${ }^{8}$ Edi- \\ tor in Chief, Chest, Respiratory Institute, Cleveland Clinic, Cleveland, OH, USA \\ ${ }^{9}$ Editor in Chief, Annals of Intensive Care, Service de Médecine Intensive-Réan- \\ imation, Hôpital Bicêtre, AP-HP Université Paris-Saclay, Le Kremlin-Bicêtre, \\ France. ${ }^{10}$ Editor in Chief, Critical Care, Department of Intensive Care, Erasme \\ University Hospital, Université Libre de Bruxelles, Bruxelles, Belgium. ${ }^{11}$ Past \\ Editor in Chief, Intensive Care Medicine, Médecine Intensive Et Réanimation, \\ APHP, Hôpital Saint-Louis, Paris University, Paris, France.
}

\section{Author contributions}

EA, GC: Conception of the work, All: Drafting of the article, All: Critical revision of the article, All: Final approval of the version to be published.

\section{Compliance with ethical standards}

\section{Conflicts of interest}

LB's laboratory has received research grants or equipment from Medtronic Covidien, Fisher Paykel, Air Liquide, Philips, Sentec, and has a patent with General Electric. Dr. Jaber has received consulting fees from Drager, Medtronic, Baxter, Fresenius Medical and Fisher and Paykel. GC has received consulting fees and institutional research grants from Neuroptics and Integra. The other authors declare no competing interests for this manuscript.

\section{Publisher's Note}

Springer Nature remains neutral with regard to jurisdictional claims in published maps and institutional affiliations.

Received: 2 June 2020 Accepted: 8 June 2020

Published online: 6 July 2020

\section{References}

1. Sharing research data and findings relevant to the novel coronavirus (COVID-19) outbreak. https://wellcome.ac.uk/coronavirus-covid-19/opendata. Accessed 30 May 2020

2. Retracted coronavirus (COVID-19) papers https://retractionwatch.com/ retracted-coronavirus-covid-19-papers/. Accessed 30 May 2020

3. Glasziou PP, Sanders S, Hoffmann T. Waste in covid-19 research. BMJ. 2020 May 12;369:m1847. https://doi.org/10.1136/bmj.m1847. PMID: 32398241. 